\title{
Chemical tethering of motile bacteria to silicon surfaces
}

\author{
Jane P. Bearinger ${ }^{1}$, Lawrence C. Dugan ${ }^{2}$, Ligang Wu², Haley Hill ${ }^{3}$, Allen T. Christian ${ }^{4}$, and Jeffrey A. Hubbell ${ }^{5}$ \\ ${ }^{1}$ Lawrence Livermore National Laboratory, Applied Physics and Biophysics, Livermore, CA, USA, ${ }^{2}$ Lawrence \\ Livermore National Laboratory, CMELS, Livermore, CA, USA, ${ }^{3}$ Department of Chemistry, Northwestern \\ University, Evanston, IL, USA, ${ }^{4}$ Monsanto, St. Louis, MO, USA, and the Institute of Bioengineering and Institute \\ of Chemical Sciences and Engineering, École Polytechnique Fédérale de Lausanne (EPFL), Lausanne, Switzerland
}

BioTechniques 46:209-216 (March 2009) doi 10.2144/000113073

Keywords: E. coli; tethering; patterning; microarrays; imaging; host-pathogen response; drug discovery; biofuel cells Supplementary material for this article is available at www.BioTechniques.com/article/113073.

We chemically immobilized live, motile Escherichia coli on micrometer-scale, photocatalytically patterned silicon surfaces via amine- and carboxylic acid-based chemistries. Immobilization facilitated ( $i$ ) controlled positioning; (ii) high resolution cell wall imaging via atomic force microscopy (AFM); and (iii) chemical analysis with time-of-flightsecondary ion mass spectrometry (ToF-SIMS). Spinning motion of tethered bacteria, captured with fast-acquisition video, proved microbe viability. We expect our protocols to open new experimental doors for basic and applied studies of microorganisms, from host-pathogen relationships, to microbial forensics and drug discovery, to biosensors and biofuel cell optimization.

\section{Introduction}

Biomolecular placement with controlled spatial resolution is of significant interest to expanding research programs in $(i)$ host-pathogen biology, (ii) biosensors, (iii) and biofuel cells. (i) Pathogen study requires appropriate placement and containment protocols. While micro-injection (1) is a well-controlled technique used by some investigators to deliver pathogens into hosts, more common techniques, such as incubating (2), are not well controlled and pathogen release-whether triggered by accident or intention-can have deleterious health effects. (ii) Biosensors require extremely localized chemical surface modifications to enable associated biological reagent activity, electrical transduction, and readout (3-6). Biosensor power is typically defined by the density of information on a chip: the tighter and more localized the patterning, the more powerful the chip. (iii) Microbial fuel cells (7-10) require electron transduction from the organism to an electrode, defining a minute distance for charge to travel. Optimal signal transduction may be accomplished by engineering electron transport paths between microbe and electrode. Our procedures dictate placement and improve containment of microorganisms under investigation, thereby facilitating characterization by complementary analysis techniques.

Other groups have labeled bacteria or dictated bacterial placement. Solution- based fluorescence $(11,12)$ and quantum$\operatorname{dot}(13,14)$ labeling are two such methods that enable simple bacterial visualization. Technetium labeling (15) for medical purposes and covalent labeling of fusion proteins (16) have also been reported, and Da Silva et al. biotinylated bacteria for subsequent avidin-based interactions (17). Large-scale, low-resolution bacterial patterning-historically using replica plating $(18,19)$ and more recently with pin arraying $(20,21)$ - enables microbial genetic and biofilm formation studies. Immobilizing microbes on polymeric hydrogels $(22,23)$ can limit motility and maintain viability. Weibel et al. described the use of micropatterned agarose stamps to print patterns of bacteria on agar plates to study pattern growth and bacterial strain interactions (24). In these experiments, features as small as $200 \mu \mathrm{m}$ were printed over areas as large as $50 \mathrm{~cm}^{2}$. More recently, Keymer et al. constructed habitat microfluidics (linear arrays of coupled, micro-scale patches) to study adaptive dynamics of the bacterial metapopulations (25). Our method of high-resolution microbial patterning incorporates labeling and tethering steps, such that microbe viability is maintained, thereby facilitating analysis.

Our sample preparation protocols pattern and tether live bacteria. Once tethered, the bacteria are available for examination via a number of techniques, or can serve as instrumentation themselves. We first tested our methods by examining bacteria optically with reflectance and fluorescence microscopy, and with atomic force microscopy (AFM); these techniques facilitate in situ examination. We also tested our methods under high-vacuum conditions, with time-of-flight secondary ion mass spectometry (ToF-SIMS). We demonstrate the use of multiple examination techniques on a single sample, in order to gain corroborating information and validate the multiplexing capabilities of our protocols.

We chose Escherichia coli, a Gramnegative bacterium that resides in the gut, as a model microorganism for investigation. E. coli has flagella, each composed of a rotary motor and filament. These flagella enable a cell to swim and confound the ability to study highresolution aspects of the live microorganism in a native environment. Two similar protocols were used to reduce their movement, exploiting amine or acid chemistry on the bacteria and enabling subsequent investigation.

\section{Materials and methods}

Chemical attachment protocols

We developed two methods of chemical attachment to E. coli: the first covalently modifies outer membrane amine groups and allows for chemical and bacterial pattern overlay checking with a fluorescent intermediate, while the second modifies outer membrane carboxyl groups and directly, covalently immobilizes bacteria to silicon. E. coli 

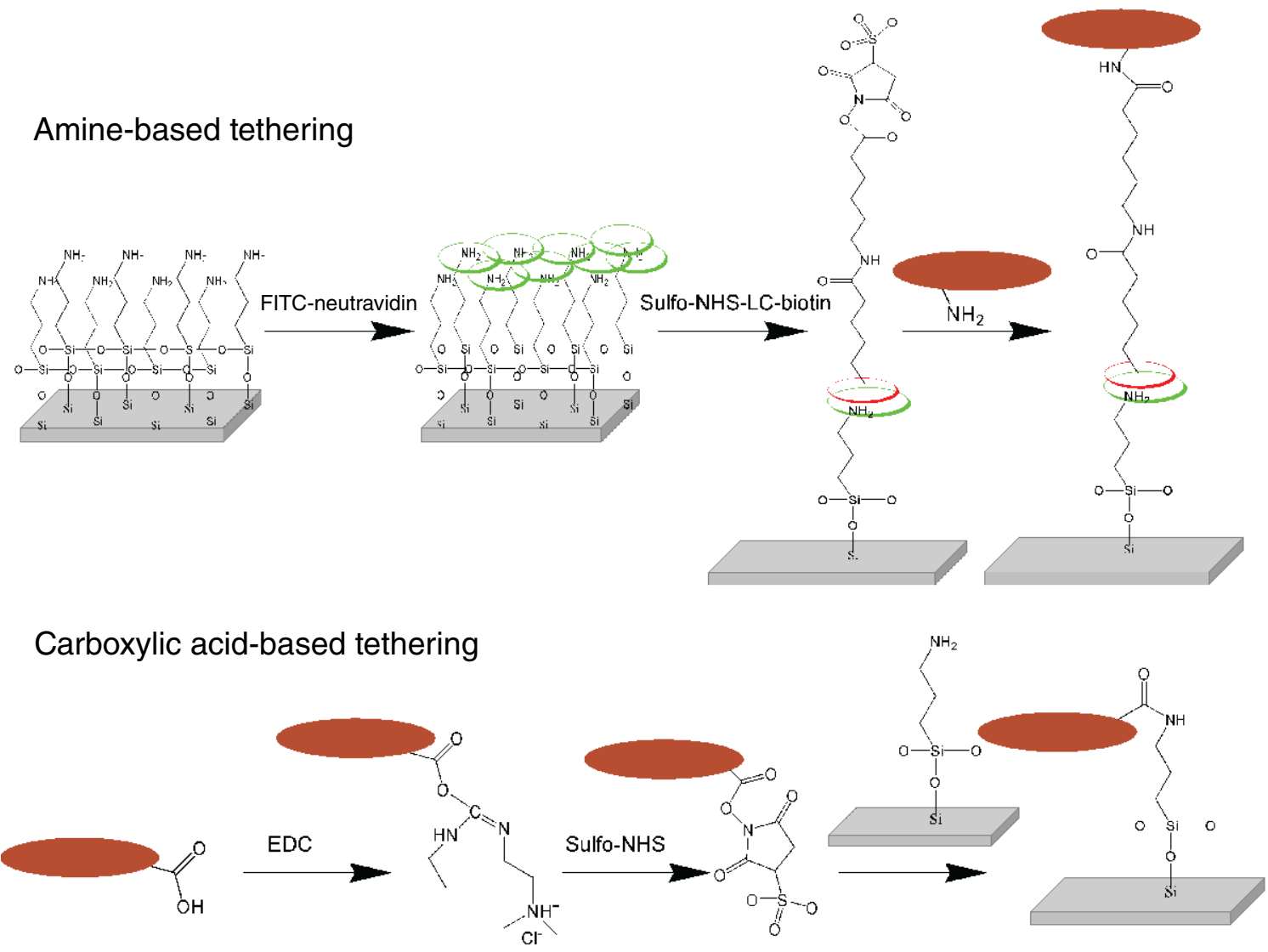

Figure 1. Schematics of amine- and carboxlic acid-based tethering protocols. Amine-based tethering of $E$. coli to substrates via fluorescent FITC-neutravidin intermediate (top) and carboxylic acid-based tethering of $E$. coli without fluorescent intermediate (bottom).

K12 MG1655 (wild-type) strain was used in all experiments. This type of E. coli has peritrichous flagella (that is, multiple flagella per bacterium that project in all directions).

Protocol 1. Amine-based attachment on patterned, non-adhesive silicon substrates, allowing intermediate fluorescent pattern visualization We generated $10 \mu \mathrm{m} \times 10 \mu \mathrm{m}$ squarepatterned regions by photocatalytically oxidizing an unsaturated silane attached to silicon by shining a red LED light (Lumex, Glenview, IL, USA) through a porphyrin-coated mask in contact with the substrate (26). Briefly, $10 \mu \mathrm{m} \times 10 \mu \mathrm{m}$ square regions of allyltrichlorosilanecoated (ATC; United Chemicals, Bristol, PA, USA) silicon substrates were oxidized away by illuminating the red LED through a porphyrin-coated mask in contact with the substrate for a few seconds, leaving a chemically patterned $\mathrm{ATC} / \mathrm{SiO}_{2}$ checkerboard. A non-fouling interpenetrating network (IPN) chemistry of P(AAm-coEG) (27) was then covalently grafted to matrix regions retaining the ATC (27). Square, bare silicon regions were then modified with aminopropyl silane (APS; United Chemicals, Bristol, PA, USA) (26).

Protein adsorbs preferentially to APS-like surfaces $(28,29)$. We incubated patterned surfaces in $25 \mu \mathrm{g} / \mathrm{mL}$ fluorescein isothiocyanate-neutravidin (FITC-neutravidin; Molecular Probes, Eugene, OR, USA) in phosphate-buffered saline (PBS, $1 \times, \mathrm{pH}$ 7.2; Sigma Aldrich, St. Louis, MO, USA) solution for $30 \mathrm{~min}$ in order to optically image our chemical pattern (26) (Figure 1). We then grafted N-hydroxysulfosuccinimide-long-chain-biotin (sulfoNHS-LC-biotin, $1 \mathrm{mg} / \mathrm{mL}$ in PBS, $\mathrm{pH}$ 8.0; Pierce, Rockford, IL, USA) to the avidin-coated $10-\mu \mathrm{m}$ squares by incubating surfaces for $30 \mathrm{~min}$.

E. coli were cultured overnight in Luria-Bertani (LB) media and grown in the presence of acridine orange $(\mathrm{AO}$; Molecular Probes), a nucleic acid-selective metachromatic stain useful for cell-cycle determination. Phase contrast microscopy demonstrated the health of the culture by confirming the presence of whole cells (i.e., no debris or ghosts). Two $1-\mathrm{mL}$ aliquots of culture were transferred into separate microcentrifuge tubes. The cells were spun for $2 \mathrm{~min}$ in an ultracentrifuge at $8,000 \times \mathrm{g}$. Cells were resuspended in $1 \mathrm{~mL}$ of ice-cold PBS. Cell spin-down, supernatant transfer, resuspension in cold PBS, and spin-down steps were repeated. Finally, cells were resuspended in $1 \mathrm{~mL}$ of room-temperature PBS and incubated on the biotin-modified, rinsed surfaces for $30 \mathrm{~min}$ to link the amine groups on the cell wall of $E$. coli to the tethers on the silicon surface (Figures 1, 2, and 3A). Tethered cells were then available for further analysis.

Protocol 2. Direct carboxylic acidbased attachment to silicon substrates Photocatalytically patterned substrates were generated by homogeneously modifying silicon with amino silane and then locally oxidizing away $10 \mu \mathrm{m} \times 10 \mu \mathrm{m}$ square regions of the silane. This resulted in squares of $\mathrm{SiO}_{2}$ surrounded by an APS matrix. Thus, in this scenario, we tethered the bacteria to the matrix, leaving bare central regions on the substrate (Figure 3B).

After cells were cultured and transferred from media to PBS, as described previously in Protocol 1, we activated 
A

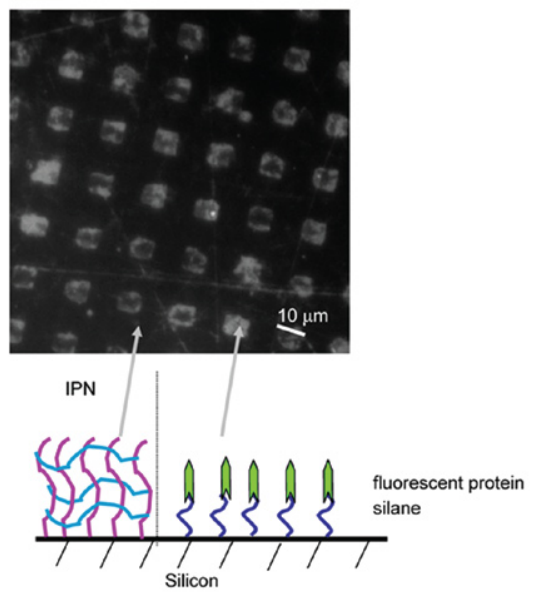

B

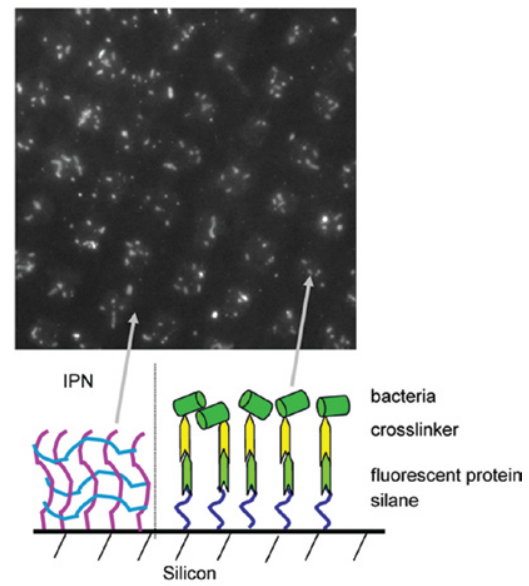

A

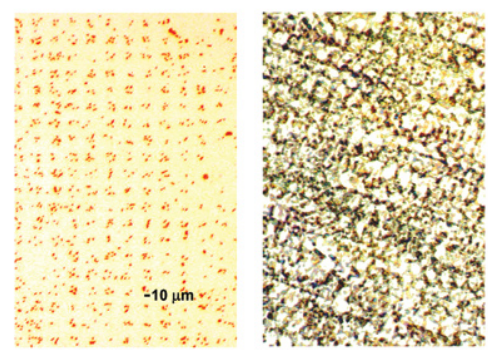

Figure 3. Reflectance images of patterned $E$ coli. Reflectance images of $E$. coli bound to squares (A) and matrices (B) following amine-based and carboxylic acid-based tethering protocols, respectively.
Figure 2. Patterning of $E$. coli onto $10-\mu \mathrm{m}$ squares via fluorescent protein intermediate. (A) Square patterns generated via photocatalytic lithography. A photomask coated with porphyrin and in contact with allyltrichlorosilane (ATC)-coated silicon, was exposed to red light for several seconds. ATC was locally oxidized away, patterning the surface. A non-fouling polymer layer was synthesized on the patterned ATC on silicon (IPN). After back-filling bare silicon regions with aminopropylsilane (APS), the substrate was incubated with a solution of fluorescein-labeled neutravidin. The fluorescence micrograph shows that protein selectively adsorbs to APS regions, and is repelled by the non-fouling polymer (20x magnification, square width $=4 \mu \mathrm{m}$ ). (B) E. coli were tethered onto the FITC-neutravidin-coated squares by linking biotin crosslinks modified with NHS esters and then exposing washed $E$. coli to the modified substrates.

functional groups directly on the bacterial membrane and then adhered them to the amine-functionalized substrates. Cells were re-suspended in 2 tubes containing $500 \mu \mathrm{L}$ of $0.1 \mathrm{M} 2$-(N-Morpholino) ethanesulfonic acid (MES buffer, $\mathrm{pH}$ 5.5; Pierce). The contents of the 2 tubes were combined, and $2.4 \mathrm{mg}$ 1-ethyl-3(3-dimethylaminopropyl)carbodiimide hydrochloride (EDC, Pierce) and 6.4 mg N-hydroxysulfosuccinimide (sulfoNHS, Pierce) were added to the tube and gently mixed on and off for $15 \mathrm{~min}$. Cells were spun down a last time at $8,000 \times g$, and supernatant was removed. Cells were resuspended in $1 \mathrm{~mL}$ of PBS and incubated with the amine-terminated substrate for $30 \mathrm{~min}$. After $30 \mathrm{~min}$, the substrate was vigorously rinsed with sterile ultrapure water (UPW, $18 \mathrm{M} \Omega \mathrm{cm}$ ) before placing in media. Tethered cells then were available for analysis via optical microscopy, AFM, and ToF-SIMS.

\section{Optical microscopy}

We detected surface-adsorbed FITClabeled neutravidin and $E$. coli bound to FITC-neutravidin (via Protocol 1) in an aqueous environment using a Zeiss AxioVert 200M materials microscope equipped with darkfield, epifluorescence, a FITC filter set, and a Zeiss Axiocam HRM high resolution digital camera (Thornwood, NY, USA). Images were captured using the Zeiss Axiovision software.

We acquired reflectance images of $E$. coli on silicon with a Nikon D100 camera
(Melville, NY, USA) mounted on a Nikon Labophot 2 microscope after the substrates had been rinsed in water and gently dried with nitrogen.

\section{Atomic force microscopy}

Imaging of E. coli tethered via Protocol 2 was performed on a Digital Instruments NanoScope IV atomic force microscope (Veeco Metrology, Inc., Santa Barbara, CA, USA) equipped with a tappingmode fluid cell. Tapping mode minimizes tip-induced effects on the outer membrane structure. Images were collected in phase mode and first-order flattened. The scan rate was approximately $2 \mathrm{~Hz}$ for the highresolution imaging of the structures. E. coli imaging was performed dry using Silicon Super Sharp Tips (SSS-NCHR-50, SiN, spring constant $10-130 \mathrm{~N} / \mathrm{m}$, tip radius 2 nm; Nanosensors, Neuchâtel, Switzerland). Images were composed of $512 \times 512$ lines, which represented $4 \mu \mathrm{m}$ per side. Bacillus atropheus imaging was performed in water using a phosphorus-doped Si Tip (Model no. DMASP, spring constant $1-5 \mathrm{~N} / \mathrm{m}$, Veeco Metrology, Inc.).

Time-of-flight secondary ion mass spectometry (ToF-SIMS)

ToF-SIMS measurements on bacteria tethered via both protocols were conducted on a PHI-TRIFT III instrument equipped with a gallium liquid metal ion gun ( $\left.\mathrm{Ga}^{+} \mathrm{LMIG}\right)$ (Physical Electronics, Chanhassen, MN, USA). The ion gun was operated at $25 \mathrm{kV}$ (unbunched mode) for high image resolution. Analyses were done utilizing $\mathrm{Ga}^{+}$ions at room temperature. ToF-SIMS measurements were conducted over a $300 \mu \mathrm{m} \times 300 \mu \mathrm{m}$ area for $10 \mathrm{~min}$. The positive mass spectra were calibrated using common hydrocarbon fragment peaks at $\mathrm{CH}_{3}{ }^{+}, \mathrm{C}_{2} \mathrm{H}_{3}{ }^{+}$, and $\mathrm{C}_{4} \mathrm{H}_{7}{ }^{+}$, while the negative mass spectra were calibrated using $\mathrm{CH}^{-}, \mathrm{OH}^{-}$, and $\mathrm{C}_{2} \mathrm{H}^{-}$. Spectra for background controls were acquired by analyzing clean silicon areas on the wafers. To highlight distribution of specific ions, images were superimposed on top of the total ion image or silicon background image in order to indicate localization of species.

\section{Results}

Exploiting amines and acids on $E$. coli To dictate bacterial spatial placement, we photocatalytically patterned a mino-silane chemistry on silicon. Amino-functionalized silicon was then exposed to $E$. coli activated to conjugate to substrates via carboxylic acids or amines on the $E$. coli wall and/or flagella. While the acid-based tethering technique is purely covalent, the amine-based protocol incorporated a standard NHS-biotin crosslink so that we could visually track pattern formation and ensuing bacterial position sequentially using fluorescence.

Figure 2A indicates fluorescent FITCneutravidin protein adsorption on $10 \mu \mathrm{m}$ $\times 10 \mu \mathrm{m}$ regions of amino-functionalized silicon, surrounded by a non-adhesive polymer-grafted matrix. The islands, which were imaged dry, indicate a degree of peripherally enhanced fluorescent signal. We attribute this edge effect to surface tension: upon drying, residual solution coalesced and concentrated around the edges of the $10-\mu \mathrm{m}$ boxes.

Figure $2 \mathrm{~B}$ reveals bacteria tethered onto the protein-coated islands, coupled by the amine-based tethering protocol. The same optics that were used to 
visualize the FITC-coated boxes were used to image the bound bacteria; bacteria fluoresced green indicating a quiescent state. We reasoned that the initial experiments should inexpensively confirm spatial mapping of substrate and bacterial patterning. Furthermore, while the biotin-avidin binding system is quite strong, one could exploit $\mathrm{pH}$ to use this system as a cleavable linkage. Thus, we expect other cleavable linkers to be useful in this regard as well, triggered by voltage, heat, or $\mathrm{pH}$.

Reflectance images of $E$. coli bound to the squares or matrix regions are shown in Figure 3. Figure 3A was acquired on the same sample shown in Figures 2 (a non-adhesive matrix and APS squares). Figure $3 \mathrm{~B}$ shows the approximate inverse surface: a photocatalytically patterned surface consisting of an amino-functionalized matrix and $\mathrm{SiO}_{2}$ squares. E. coli only tethered to substrate regions modified with amino-silane, thus patterning on the squares or matrix regions, respectively.

Figure 3B employed the carboxylic acid-based, purely covalent bacterial tethering technique. This method has also been used to investigate $B$. atropheus (see "AFM studies reveal high-resolution structure of cell membrane"). This chemical tethering technique is fast and robust, but it is more difficult to track the surface modification because there is no incorporated fluorescence.

In order to test the importance of the IPN, we did not graft the non-fouling background on substrates generated via Protocol 2. Our initial results indicate that bacterial incubation time is a key parameter in limiting non-specific binding, as bacterial incubation times of $\sim 2 \mathrm{~h}$ in the absence of the IPN indicate decreased pattern resolution.

Tethered microbes are viable $\mathrm{AO}$ staining resulted in green fluorescence, indicating that the dye had intercalated with DNA, and also indicating a more quiescent bacterial state. $\mathrm{AO}$ intercalating with RNA fluoresces red, which is indicative of an active, growth state. Although $E$. coli fluoresced green, fast image acquisition performed over a period of $1 \mathrm{~min}-1 \mathrm{~h}$ revealed spinning (reciprocating motion of some tethered bacteria), which confirmed viability. Although $1 \mathrm{~h}$ is sufficient time for binary fission to take place, we did not observe any evidence of such behavior and, therefore, do not know if manipulating the cell membrane affects such activity. We hypothesize, however, that the PBS environment precludes replication more than tethering

A

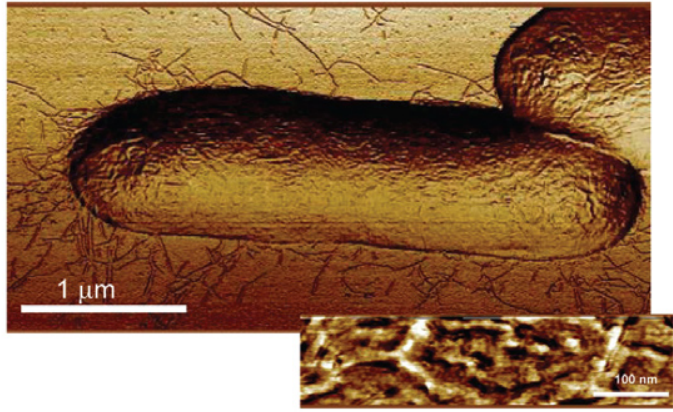

B

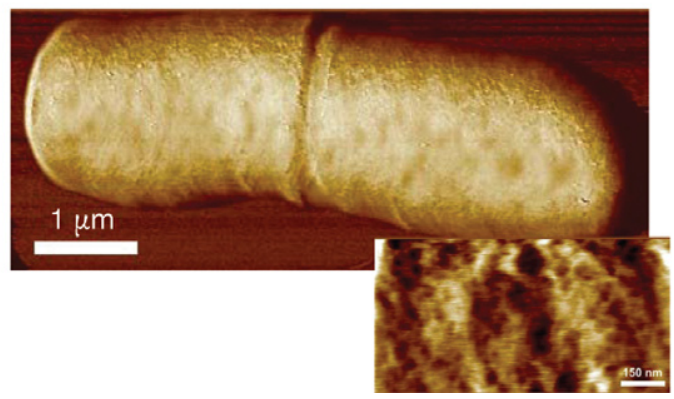

Figure 4. High-resolution tapping mode AFM images of $\boldsymbol{E}$. coli and $\boldsymbol{B}$. atropheus. High-resolution tapping mode AFM imaging of (A) E. coli (dry) and (B) B. atrophaeus (at division stage and in water) covalently tethered to silicon substrate. Figure B courtesy of A. Malkin, M. Plomp, and H. Hill. Inset figure in (A) suggests an S-layer with hexagonal symmetry. Inset figure in (B) shows topography of peptidoglycan layer.

does. Additional studies are necessary to decouple the factors.

AFM studies reveal high-resolution structure of cell membrane

Figure $4 \mathrm{~A}$ shows an image of a single tethered $E$. coli bacterium and an inset of the outer membrane (image widths of 4 $\mu \mathrm{m}$ and $500 \mathrm{~nm}$, respectively). The image was cropped in height to focus on one specific cell. The inset represents $500 \mathrm{~nm}$ width acquired as 512 lines. The images convey the flagella that enable motility and a series of interconnected ridges, elevated domains, and a complex matrix. The inset figure suggests an S layer with hexagonal symmetry. Similar, in-depth investigations into cell walls have aided the understanding of pathogen architecture and its application to microbial forensics and medicine $(23,30)$.
Figure $4 \mathrm{~B}$ shows a tethered $B$. atrophaeus bacterium and high-resolution membrane inset, courtesy of collaboration with the Malkin group. The large image was $4 \mu \mathrm{m}$ across, taken at $512 \times 512$ lines and cropped to focus on the bacterium. The inset image width is $1000 \mathrm{~nm}$, acquired as 512 lines. We included $B$. atropheus to indicate the versatility of the chemical attachment protocol and to provide initial insights into a Grampositive bacterium lacking flagellum. Gram-positive and Gram-negative bacteria have different surface architectures: peptidoglycans are present on the outerlayer of Gram-positive bacteria, while Gram-negative bacteria possess an outer membrane covered with proteins and lipopolysaccharides. It is notable that the growth/division stage of $B$. atropheus is evident in Figure 4B. However, we

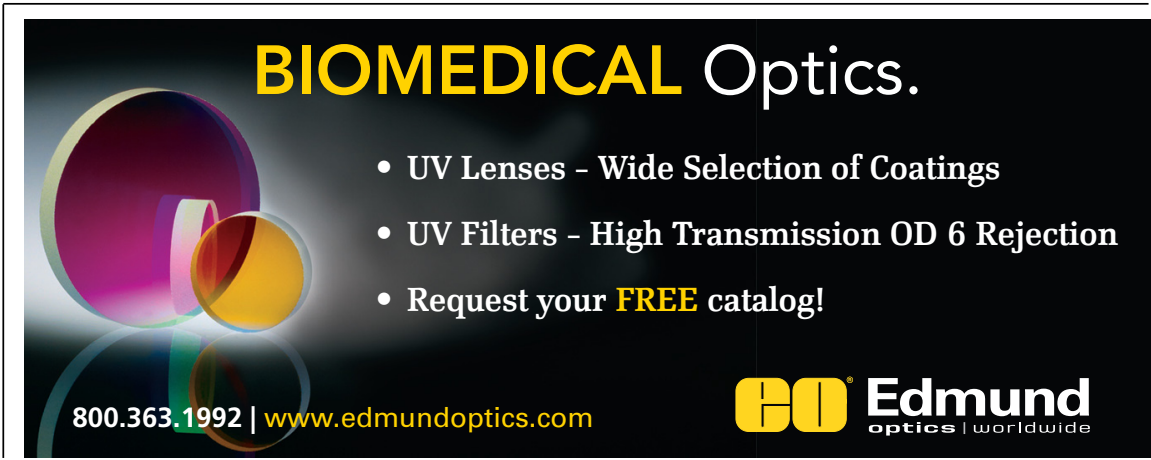


A

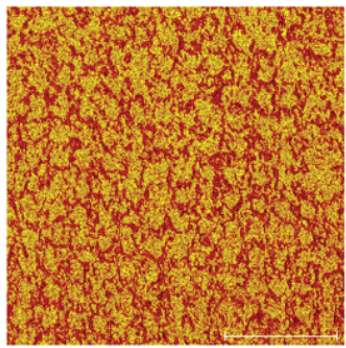

tot

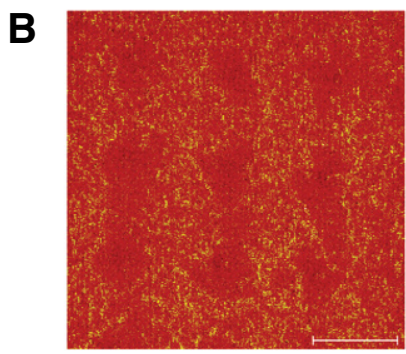

tot

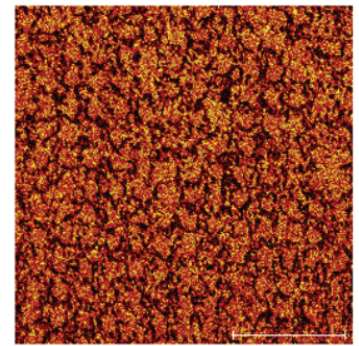

$\mathrm{Si}$

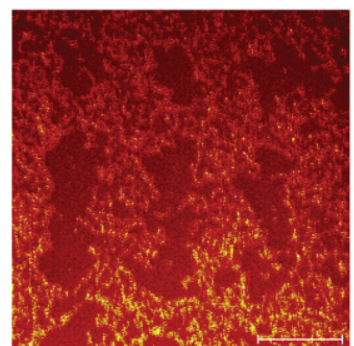

SEM

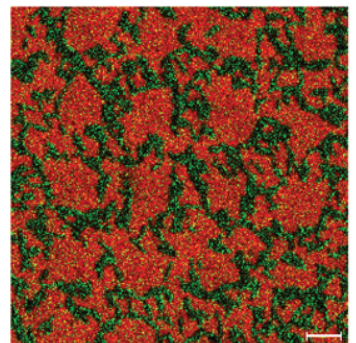

147 on $\mathrm{Si}$

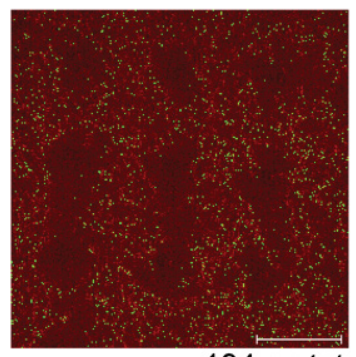

194 on tot

Figure 5. ToF-SIMS imaging of $\boldsymbol{E}$. coli. Excerpts of ToF-SIMS positive ion imaging (A) and negative ion imaging $(B)$ of $E$. coli enclosing bare silicon regions. Positive ion images include total ion (tot), $\mathrm{m} / \mathrm{z}$ ratios indicative of Silicon ( $\mathrm{Si}$ ), and overlay of $\mathrm{m} / \mathrm{z}$ indicative of a peptidoglycan constituent on the silicon image (147 on $\mathrm{Si}$ ). Negative ion images include total ion (tot), standard error of the mean (SEM), and overlay of $\mathrm{m} / \mathrm{z}$ indicative of a lipopolysaccharide outer membrane constituent on the total ion image (194 on tot).

are presently unable to determine if division began before or after tethering commenced.

\section{ToF-SIMS for forensic applications}

ToF-SIMS performed on a homogeneous population of cells is useful for identifying bacterial location and chemical correlation in order to develop protocols for future work targeting the identification of a random sample of bacteria. We subjected patterned bacterial samples to high-vacuum conditions and characterized them with ToF-SIMS. Although we expect ToF-SIMS (and MALDI) to be useful in future drug discovery platforms, ToF-SIMS is already used in microbial forensics: for example, to distinguish Bacillus species (31).

Highlights of the positive- and negative-mode imaging ToF-SIMS experiments are shown in Figure 5. The positive ion imaging (top) reveals the same matrixbound $E$. coli pattern imaged optically in Figure 3B attached via Protocol 2. In both the total and $\mathrm{Si}^{+}$ion [mass-tocharge ratio $(\mathrm{m} / \mathrm{z})$ of 28$]$ images, the $E$. coli regions are darker than the silicon substrate, indicating less ion release than on the substrate. However, the superimposition of $\mathrm{m} / \mathrm{z}$ 147-thought to be associated with protonated lysine [a peptidoglycan constituent $(32,33)]$ - on the silicon image reveals specific association of the peak with the bacteria. Similarly,

superimposition of $\mathrm{m} / \mathrm{z} 355$, thought to be a fragment of cardiolipin (31), also reveals association with the bacteria. The acylium ion of esterified tetratridecanoic acid, previously characterized with $\mathrm{m} / \mathrm{z}$ of 205 (31), also associated with the bacterial regions (data not shown).

The lower portion of Figure 5 highlights the negative ion imaging of $E$. coli attached via Protocol 1. In this experiment, a different patterning template was used, rendering bacterial attachment around $40-\mu \mathrm{m}$ diameter bare $\mathrm{SiO}_{2}$ circles. Total ion and SEM images indicate the dense matrix of $E$. coli surrounding the silicon circles. Superposition of $\mathrm{m} / \mathrm{z} 194$ on the total ion image indicates a decanoic acid (myristic acid) derivative (34), preferentially associated with the bacteria. $\mathrm{m} / \mathrm{z}$ values of 227,255 , and $283-$ also associated with lipopolysaccharide outer membrane constituents (34)-again associate with the bacterial regions.

\section{Discussion}

Bacteria sense chemicals, and rely on their hardwired chemical sensors (chemoreceptors) to determine what materials they need and should swim toward, and what materials are toxic and from which they should swim away. They swim with the assistance of their flagella, each attached to a rotary motor that is embedded in the cell wall (35). Restraining the flagella so that they cannot turn causes the cell body to turn.

We infer from acquired video (see Supplementary Movie 1) that the motion of our tethered bacteria is related to the number of chemical tethers. We hypothesize that numerous chemical tethers along the length of bacteria and flagella lock the organism to the substrate, whereas fewer chemical tethers enable limited motion. The reciprocating motion is described as spin. Future work may examine the strength of the tethers with force microscopy or other appropriate techniques to quantitatively determine tether strength and tether multiplicity. We envision our construct to be useful in environmental sensing and remediation, perhaps in conjunction with colorimetric or light-emitting readout.

Acquired video of $E$. coli tethered to APS-coated substrates confirmed organism viability but did not reveal any evidence of tethered bacteria fission. Fission requires a number of biochemical reactions and events; among them is growth of the prokaryotic cell wall. Prokaryote cell wall structure and surface chemistry are not well understood. While investigation with molecular biology studies of deletion mutants (36,37), optical microscopy $(38,39)$, and cryo-electron microscopy (40-42) studies provide information, all these techniques have shortcomings. Sample preparation harms the specimen (temperature, necessity of vacuum, etc.), and the sample is distorted. In contrast, AFM allows examination of live structures to gain structural information, for both structure-function relationships and microbial forensics.

Previously, gelatin $(22,23)$ has been used to limit prokaryote motion. While trapping bacteria in gelatin has proven easy and effective, the bacteria typically sink down into the gelatin, leaving only part of their cell walls available for investigation. Furthermore, embedding in gelatin precludes further manipulation or chemical study.

Our novel methods facilitate AFM subsequent to optical microscopy, in order to image prokaryotic cells with nanometer resolution in liquid in tapping mode without the use of gelatin. In tapping mode AFM (TM-AFM), the cantilever oscillates at its resonance frequency, such that the tip moves in and out of contact with the sample as the piezoelectric scanner moves the sample in the $\mathrm{X}$ and $\mathrm{Y}$ directions. TM-AFM is useful for imaging soft and/or delicate biological materials, while retaining fine structure. Our robust tethering techniques prevented the cells 
from swimming away while under investigation.

In many bacteria, in the outermost component of the cell envelope, biological material comprises a two-dimensional crystalline array known as a surface layer, or S-layer. S-layers are composed of identical protein or glycoprotein subunits, which are linked non-covalently to each other and to the underlying cell envelope. The regular, high-density arrangement of functional groups, combined with the ability to self-assemble in suspension and recrystallize on a solid support, makes $\mathrm{S}$-layers (such as those visualized in Figure 4A) prime candidates for biotechnological manipulation.

The capacity to view S-layers on motile bacteria via high-resolution AFM may also provide an approach by which to study the mechanisms behind bacterial capsule loss, a phenomenon whereby a cell coat changes from smooth to rough on specific bacteria, typically accompanied by a decrease in cell virulence. We also envision a drug discovery platform that examines real-time changes to the cell coat upon chemical additions to the media in a fluid cell AFM environment. Possible enhancements to the technique may lead to microbial tethering for cellcycle studies relevant to medical and hostpathogen studies.

While we concentrated on APS-coated substrates for bacterial tethering in AFM and all other experiments, we plated activated bacteria on silicon, IPN-coated silicon, and APS-coated silicon substrates for $30 \mathrm{~min}-1 \mathrm{~h}$. After $1 \mathrm{~h}$ of plating E. coli on clean silicon and IPN-coated silicon, only a few aberrant bacteria attached to substrates. In contrast, relatively dense bacterial adhesion was seen on APS surfaces. These varying surfaces therefore provided our positive and negative controls.

Clean silicon substrates exposed to bacteria for $\sim 2 \mathrm{~h}$ showed increased non-specific binding. Cao et al. (43) reported that native properties of the individual strain of bacteria and the specific functional structure of surfaces determine the strength of force interactions, and the extent of adhesion. This is consistent with our findings, so we did not conduct a more in-depth study on the kinetics of nonspecific binding on our three surfaces. While it may be relevant for $E$. coli, it would not be relevant for $B$. atropheus, or other species.

We used APS chemistry to accomplish tethering because it is commonly used to introduce terminal amine groups to oxide surfaces. However, Wang et al.
(44) reported compromised stability of APS self-assembled layers when exposed to saline solution at body temperature for 10 days. While we only maintained tethered bacterial surfaces in solution at room temperature for a few hours at a time, we acknowledge longer-range goals of (i) substrate or device storage and (ii) biofuel cell use, both of which may be problematic based on such findings. We therefore point out that glycidyl-terminated silanes (44) may prove to be of greater utility for applications requiring long-term stability.

As an addendum to this work, we envision tethering dense fields of bacteria around small regions in which minute drug volumes could be specifically placed, in a manner that allows tracking and data acquisition in the absence of a technician. In many cases, the same substrate, as indicated in these experiments, could be used with various techniques of interest, corroborating and enriching the data pool. Information could be collected on oxidative stress state, vegetative versus spore state, as well as what compounds affect peptidoglycan assembly, crystallinity, etc.

Another possible extension of our platform includes its use as an alternative energy fuel-cell platform. Alternative energy investigations based on microbial fuel cells began in 1962 (8,45$50)$. Bacteria are typically placed in chambers, on a membrane, or in a gel (51) in close contact with electrode pads, as electron transfer through biofilms to electrodes must take place. Microbial fuel cells to date have achieved only limited efficiency because of the low output that is observed. One study, aimed at denitrification, linked bacteria to electroconductive polymers via avidin-biotin bonds, but the avidin was purported to reduce bacterial activity (17). We envision that slight modifications to our covalent tethering system-such as tethering via conjugated carbon chains - could be used to position cells at specific distances from an anode. Controlled bacterial charge transport through conductive tethers and neighboring bacterial pili, through which current can travel as a "nanowire" (52), should increase electron conversion, thereby increasing system efficiency.

\section{Conclusion}

We developed robust and reproducible methods for attaching live bacterial cells to surfaces suitable for optical and atomic force microscopy imaging under native conditions, and for chemical analysis techniques with or without high vacuum. Our protocols are compatible with a variety of substrates and biomolecules, and we believe these techniques will impact forensic, drug discovery and biofuel cell technologies.

\section{Acknowledgements}

We gratefully acknowledge funding from the National Institutes of Health (NIH; grant no. R21 EB003991 and from the Laboratory Science and Technology Office (LSTO; grant no. 03-ERD-068). We also acknowledge support from the National Science Foundation's Center for Biophotonic Science and Technology at the University of California, Davis. This work was partially performed under the auspices of the U.S. Department of Energy by Lawrence Livermore National Laboratory under contract W-7405-Eng-48 and under Contract DE-AC52-07NA27344. We thank K. Michlitsch, J. Poco, J. Reynolds, A. Hiddessen and A. Hinz for their efforts and insights. This paper is subject to the NIH Public Access Policy.

Authors J.B. and J.H. are listed as co-inventors for a patent (USPTO application no. 20060127595) related to the patterning technique used in this publication. All other authors declare no competing interests.

\section{References}

1. Goetz, M., A. Bubert, G.F. Wang, I. ChicoCalero, J.A. Vazquez-Boland, M. Beck, J. Slaghuis, A.A. Szalay, and W. Goebel. 2001. Microinjection and growth of bacteria in the cytosol of mammalian host cells. Proc. Natl. Acad. Sci. USA 98:12221-12226.

2. Kesty, N.C., K.M. Mason, M. Reedy, S.E. Miller, and M.J. Kuehn. 2004. Enterotoxigenic Escherichia coli vesicles target toxin delivery into mammalian cells. EMBO J. 23:4538-4549.

3. Mendes, P.M., C.L. Yeung, and J.A. Preece. 2007. Bio-nanopatterning of surfaces. Nanoscale Res. Lett. 2:373-384.

4. Conway, B.R. and K.T. Demarest. 2002. The use of biosensors to study GPCR function: applications for high-content screening. Receptors Channels 8:331-341.

5. Harwood, G.W. and C.W. Pouton. 1996 Amperometric enzyme biosensors for the analysis of drugs and metabolites. Adv. Drug Deliv. Rev. 18:163-191.

6. Davis, F. and S.P.J. Higson. 2005. Structured thin films as functional components within biosensors. Biosens. Bioelectron. 21:1-20.

7. Debabov, V.G. 2008. Electricity from microorganisms. Microbiology 77:123-131.

8. Davis, F. and S.P.J. Higson. 2007. Biofuel cells--Recent advances and applications. Biosens. Bioelectron. 22:1224-1235.

9. Rismani-Yazdi, H., S.M.Carver, A.D. Christy, and O.H. Tuovinen. 2008. Cathodic limitations in microbial fuel cells: an overview. J. Power Sources 180:683-694. 
10. Rodriguez, J.,J.M. Lema, and R. Kleerebezem. 2008. Energy-based models for environmental biotechnology. Trends Biotechnol. 26:366374.

11. Porte, F., J.P. Liautard, and S. Kohler. 1999. Early acidification of phagosomes containing Brucella suis is essential for intracellular survival in murine macrophages. Infect. Immun. 67:4041-4047.

12. Agerer, F., S. Waeckerle, and C.R. Hauck. 2004. Microscopic quantification of bacterial invasion by a novel antibody-independent staining method. J. Microbiol. Methods 59:23-32.

13. Kloepfer, J.A., R.E. Mielke, and J.L. Nadeau. 2005. Uptake of CdSe and CdSe/ZnS quantum dots into bacteria via purine-dependent mechanisms. Appl. Environ. Microbiol. 71:2548-2557.

14. Kloepfer, J.A., R.E. Mielke, M.S. Wong, K.H. Nealson, G. Stucky, and J.L. Nadeau. 2003. Quantum dots as strain- and metabolismspecific microbiological labels. Appl. Environ. Microbiol. 69:4205-4213.

15. Johanson, W.G., M.G. Kennedy, and F.J. Bonte. 1973. Use of Technetium (Tc-99m) as a Bacterial Label in Lung Clearance Studies. Appl. Microbiol. 25:592-594.

16. Keppler, A., S. Gendreizig, T. Gronemeyer, H. Pick, H. Vogel, and K. Johnsson. 2003. A general method for the covalent labeling of fusion proteins with small molecules in vivo. Nat. Biotechnol. 21:86-89.

17. DaSilva, S., L. Grosjean, N. Ternan, P. Mailley, T. Livache, and S. Cosnier. 2004. Biotinylated polypyrrole films: an easy electrochemical approach for the reagentless immobilization of bacteria on electrode surfaces. Bioelectrochem. Proc.XVII Inter. Symp. Bioelectrochem. Bioen. 63:297-301

18. Lederberg, J. and E.M. Lederberg. 1952. Replica plating and indirect selection of bacterial mutants. J. Bacteriol. 63:399-406.

19. Lederberg, J. 1989. Replica plating and indirect selection of bacterial mutants: isolation of preadaptive mutants in bacteria by sib selection. Genetics 121:395-399.

20. Minas, W., J.E. Bailey and W. Duetz. 2000. Streptomycetes in micro-cultures: Growth production of secondary metabolites, and storage and retrieval in the 96-well format. Anton. Leeuw. 78:297-305.

21. Duetz, W.A., L. Ruedi, R. Hermann, K. O'Connor, J. Buchs, and B. Witholt. 2000. Methods for intense aeration, growth, storage, and replication of bacterial strains in microtiter plates. Appl. Environ. Microbiol. 66:26412646.

22. Petre, M., G. Zarnea, P. Adrian, and E. Gheorghiu. 1999. Biodegradation and bioconversion of cellulose wastes using bacterial and fungal cells immobilized in radiopolymerized hydrogels. Resour. Conserv. Recy. 27:309-332.

23. Plomp, M., T.J. Leighton, K.E. Wheeler, H.D. Hill, and A.J. Malkin. 2007. In vitro high-resolution structural dynamics of single germinating bacterial spores. Proc. Natl. Acad. Sci. USA 104:9644-9649.

24. Weibel, D.B., A. Lee, M. Mayer, S.F. Brady, D. Bruzewicz, J. Yang, W.R. DiLuzio, J. Clardy, and G.M. Whitesides. 2005. Bacterial printing press that regenerates its ink: contact-printing bacteria using hydrogel stamps. Langmuir 21:6436-6442.
25. Keymer, J.E., P. Galajda, C. Muldoon, S. Park, and R.H. Austin. 2006. Bacterial metapopulations in nanofabricated landscapes. Proc. Natl. Acad. Sci. USA 103:17290-17295.

26. Bearinger, J.P., G. Stone, A.T. Christian, L.C. Dugan, A.L. Hiddessen, K.J.J. Wu, L. Wu, J. Hamilton, et al. 2008. Porphyrinbased photocatalytic lithography. Langmuir 24:5179-5184.

27. Bearinger, J.P., D.G. Castner, S.L. Golledge, A. Rezania, S. Hubchak, and K.E. Healy. 1997. $\mathrm{P}$ (AAm-co-EG) interpenetrating polymer networks grafted to oxide surfaces: surface characterization, protein adsorption, and cell detachment studies. Langmuir 13:51755183.

28. McFarland, C.D., C.H. Thomas, C. DeFilippis, J.G. Steele, and K.E. Healy. 2000. Protein adsorption and cell attachment to patterned surfaces. J. Biomed. Mater. Res. 49:200-210

29. Thomas, C.H., C.D.McFarland, M.L.Jenkins, A. Rezania, J.G. Steele, and K.E. Healy. 1997. The role of vitronectin in the attachment and spatial distribution of bone-derived cells on materials with patterned surface chemistry. J. Biomed. Mater. Res. 37:81-93.

30. Plomp, M., T.J. Leighton, K.E. Wheeler, M.E. Pitesky, and A.J. Malkin. 2005. Bacillus atrophaeus outer spore coat assembly and ultrastructure. Langmuir 21:10710-10716.

31. Thompson, C.E., H.Jungnickel, N.P. Lockyer, G.M. Stephens, and J.C. Vickerman. 2004. ToF-SIMS studies as a tool to discriminate between spores and vegetative cells of bacteria. Appl. Surf. Sci. 231-232:420-423.

32. Satyanarayana, S., J.S. Grossert, S.F. Lee, and R.L. White. 2001. Determination of diamino acids in peptidoglycans from anaerobic bacteria. Amino Acids 21:221-235.

33. Gotschlich, E.C., B.A. Fraser, O. Nishimura, J.B. Robbins, and T.Y. Liu. 1981. Lipid on capsular polysaccharides of Gram-negative bacteria. J. Biol. Chem. 256:8915-8921.

34. Shimomura, O., F.H. Johnson, and H. Morise. 1974. Aldehyde content of luminous bacteria and of an aldehydeless dark mutant. Proc. Natl. Acad. Sci. USA 71:4666-4669.

35. Schiller, R. 1976. Bacterial chemotaxis: a survey. Gen. Relativ. Gravit. 7:127-133.

36. Mazurkiewicz, P., C.M. Tang, C. Boone, and D.W. Holden. 2006. Signature-tagged mutagenesis: barcoding mutants for genomewide screens. Nat. Rev. Genet. 7:929-939.

37. Klasson, L. and S.G.E. Andersson. 2004. Evolution of minimal-gene-sets in hostdependent bacteria. Trends Microbiol. 12:3743.

38. Vollmer, W. 2006. The prokaryotic cytoskeleton: a putative target for inhibitors and antibiotics? Appl. Microbiol. Biotechnol. 73:37-47.

39. Bhavsar, A.P. and E.D. Brown. 2006. Cell wall assembly in Bacillus subtilis: how spirals and spaces challenge paradigms. Mol. Microbiol. 60:1077-1090.

40. Craig, L., N. Volkmann, A.S. Arvai, M.E. Pique, M. Yeager, E.H. Egelman, and J.A. Tainer. 2006. Type IV pilus structure by cryo-electron microscopy and crystallography: Implications for pilus assembly and functions. Mol. Cell 23:651-662.

41. Trachtenberg, S. and S. Cohen-Krausz. 2006. The archaeabacterial flagellar filament: a bacterial propeller with a pilus-like structure. J. Mol. Microbiol. Biotechnol. 11:208-220.

42. Matias, V.R.F. and T.J. Beveridge. 2005. Cryoelectron microscopy reveals native polymeric cell wall structure in Bacillus subtilis 168 and the existence of a periplasmic space. Mol. Microbiol. 56:240-251.

43. Cao, T., H.Y. Tang, X.M. Liang, A.F. Wang, G.W. Auner, S.O. Salley, and K.Y.S. Ng. 2006 Nanoscale investigation on adhesion of $E$. coli to surface modified silicone using atomic force microscopy. Biotechnol. Bioeng. 94:167-176.

44. Wang, A., H. Tang, T. Cao, S.O. Salley, and K.Y.S. Ng. 2005. In vitro stability study of organosilane self-assemble monolayers and multilayers. J. Colloid Interface Sci. 291:438447.

45. Davis, J.B. and H.F. Yarbrough. 1962. Preliminary experiments on a microbial fuel cell. Science 137:615-616.

46. Chaudhuri, S.K. and D.R. Lovley. 2003. Electricity generation by direct oxidation of glucose in mediatorless microbial fuel cells. Nat. Biotechnol. 21:1229-1232.

47. Tender, L.M., C.E. Reimers, H.A. Stecher, D.E. Holmes, D.R. Bond, D.A. Lowy, K. Pilobello, S.J. Fertig, and D.R. Lovley. 2002. Harnessing microbially generated power on the seafloor. Nat. Biotechnol. 20:821-825.

48. Rittmann, B.E. 2006. The membrane biofilm reactor: the natural partnership of membranes and biofilm. Water Sci. Technol. 53:219-225.

49. Rismani-Yazdi, H., A.D. Christy, B.A Dehority, M. Morrison, Z. Yu, and O.H. Tuovinen. 2007. Electricity generation from cellulose by rumen microorganisms in microbial fuel cells. Biotechnol. Bioeng. 97:1398-1407.

50. Zhang, E., W. Xu, G. Diao, and C. Shuang. 2006. Electricity generation from acetate and glucose by sedimentary bacterium attached to electrode in microbial-anode fuel cells. J. Power Sources 161:820-825.

51. Matsunaga, T., I.Karube, and S. Suzuki. 1980. Some observations on immobilized hydrogenproducing bacteria: behavior of hydrogen in gel membranes. Biotechnol. Bioeng. 22:26072615.

52. Reguera, G., K.P. Nevin, J.S. Nicoll, S.F. Covalla, T.L. Woodard, and D.R. Lovley. 2006. Biofilm and nanowire production leads to increased current in Geobacter sulfurreducens fuel cells. Appl. Environ. Microbiol. 72:73457348.

Received 8 August 2008; accepted 19 November 2008.

Address correspondence to Jane P. Bearinger, Lawrence Livermore National Laboratory, Applied Physics and Biophysics, 7000 East Ave., L-211, Livermore, CA 94550. e-mail bearinger1@llnl.gov 